\title{
Photon Antibunching Proves Emission from a Single Subunit in the Autofluorescent Protein DsRed
}

\author{
Gabriel Sánchez-Mosteiro, Majolein Koopman, \\ Erik M. H. P. van Dijk, Jordi Hernando, \\ Niek F. van Hulst, and María F. García-Parajó ${ }^{*[a]}$
}

Autofluorescent proteins, in particular the well-known green fluorescent protein (GFP) have revolutionized the field of fluorescence imaging in molecular and cell biology, providing researchers with a direct and unique view into the dynamic organization and protein-protein interactions that occur in living cells. ${ }^{[1]}$ Spectrally distinct blue-shifted and red-shifted GFP mutants have been developed to facilitate multicolor and fluorescence resonance energy transfer (FRET) experiments, although the most red-shifted mutant of the wild-type GFP has its emission maximum at $529 \mathrm{~nm}$, where the influence of cellular autofluorescence is still significant. ${ }^{[1]}$ A more recently discovered protein called DsRed from the Discosoma genus of coral ${ }^{[2]}$ represents an excellent candidate for multicolor labeling and as an acceptor in a FRET pair, together with GFP mutants. DsRed exhibits emission at $583 \mathrm{~nm}$ with high fluorescence quantum yield and reduced photobleaching. ${ }^{[3]}$ Unfortunately, oligomerization of the wild-type (wt)-DsRed occurs both in vitro and in vivo, ${ }^{[3]}$ a drawback that prevents DsRed from reaching the same level of utility as the GFP variants. Although the monomer of DsRed has been obtained recently by stepwise directed

[a] G. Sánchez-Mosteiro, M. Koopman, E. M. H. P. van Dijk, Dr. J. Hernando, Prof. Dr. N. F. van Hulst, Dr. M. F. García-Parajó

Applied Optics Group, Faculty of Science \& Technology and

$\mathrm{MESA}^{+}$Institute for Nanotechnology, University of Twente,

P. O. Box 217, 7500 AE Enschede (Netherlands)

Fax: $(+31) 53-489-3511$

E-mail:m.f.garciaparajo@tn.utwente.nl 
evolution from the tetramer, its photophysical properties are comparatively poor. ${ }^{[4]}$

Ensemble and single-molecule spectroscopy (SMS) experiments $s^{[3,5-7]}$ as well as the crystal structure ${ }^{[8,9]}$ of wt-DsRed have confirmed its tetrameric nature (see Figure 1). Despite the oligomeric character of the protein, when investigated at the in-
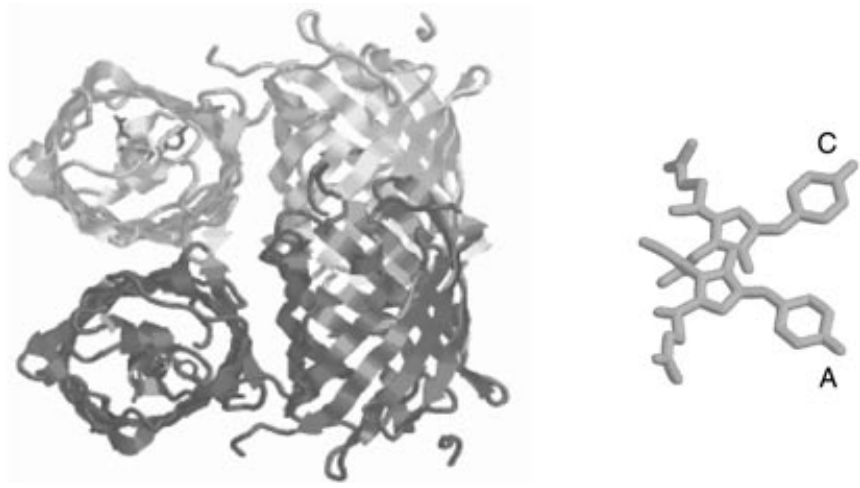

Figure 1. Schematic structure of DsRed. Ribbon diagram (left) and relative orientation of the four chromophores (right). Distance between the monomer pairs: $A-B$ and $C-D$ is $2.2 \mathrm{~nm}, A-C$ and $B-D$ is $3.8 \mathrm{~nm}$ and $A-D$ and $B-C$ is $4.3 \mathrm{~nm}$. The angular orientations between the chromophore pairs are: $21^{\circ}$ between $A-B$ and $C-D, 47^{\circ}$ between $A-C$ and $B-D$ and $41^{\circ}$ between $A-D$ and $B-C$.

dividual level, the DsRed emission exhibits blinking and discrete intensity steps, which are characteristic for a single molecule. ${ }^{[5-7]}$ Although a model that involves coupling between the four chromophores in the DsRed has been proposed, ${ }^{[6-7]}$ experimental confirmation is still lacking. In this Communication we present photon-antibunching experiments at room temperature performed on individual DsRed tetramers, which reveal that emission from DsRed proceeds dominantly from only one emitter at a given time. The results are interpreted in terms of singlet-singlet annihilation and provide clear proof that excitonic coupling dominates DsRed emission.

Photon antibunching is a clear signature of a nonclassical radiation field, which reflects the fact that a quantum system cannot spontaneously emit two photons successively without some time lag to allow the chromophore to cycle back to its excited state. ${ }^{[10]}$ Photon antibunching has been measured at cryogenic and room temperature, under continuous- or pulsed-laser illumination for individual molecules, by measuring the interphoton arrival times. ${ }^{[11-14]}$ More recently, photonantibunching has been applied to synthetic multichromophoric systems. ${ }^{[15-18]}$ Antibunching in those systems results from dipole-dipole interactions between chromophores (coherent coupling or incoherent Förster-type energy transfer), providing unambiguous evidence for singlet-singlet annihilation. ${ }^{[15-17]}$ We have exploited this concept to investigate the naturally occurring multichromophoric system DsRed.

The experiments were performed using a scanning confocal microscope with single-molecule detection sensitivity. ${ }^{[19]}$ Light from a pulsed laser source $(568 \mathrm{~nm}$ wavelength, $8 \mathrm{MHz}$ repetition rate, $0.24 \mathrm{ps}$ pulse length, $3.6 \mathrm{~kW} \mathrm{~cm}^{-2}$ average power density) was directed to the microscope and focused on the sample plane using a 1.4 NA oil-immersion objective. The emit-

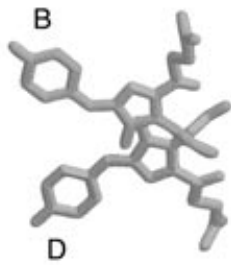

ted fluorescence from individual DsRed tetramers was split by a 50/50 nonpolarizing beam splitter and led towards two (start and stop) avalanche photodiodes, according to the classical Hanbury-Brown and Twiss coincidence experiment. ${ }^{[20]}$ The delay time between start and stop signals (i.e., between consecutive photons) was acquired using time-correlated singlephoton counting (TCSPC). From the TCSPC data, histograms of the interphoton times (coincidences) were built for a time window of $400 \mathrm{~ns}$. Given the pulsed excitation, coincidences in the histogram concentrate at discrete multiples of $T$, where $T$ is the time between two consecutive pulses (125 ns). Samples for single molecule experiments were prepared by immobilizing the proteins $\left(10^{-10} \mathrm{M}\right)$ in waterfilled pores of poly(acrylamide) gels. ${ }^{[6]}$ Only fresh samples were used in all the experiments (prepared within a day).

Figure 2 depicts a characteristic emission trajectory of DsRed. Four different levels can be distinguished in the trace, due to the progressive photobleaching of the four chromophores in the tetramer. The difference in step size for the signal intensity follows a ratio $4: 2.6: 1.2: 0.8$, instead of $4: 3: 2: 1$ as expected for equidistant intensity levels, in nice agreement with our previ-

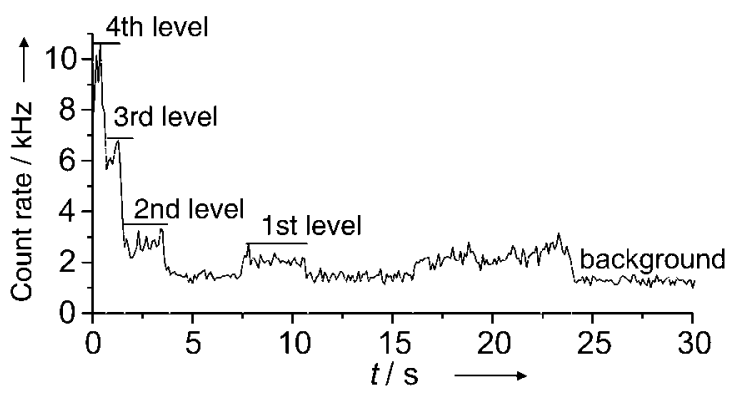

Figure 2. Real-time fluorescence intensity trajectory of the DsRed. Four intensity levels are distinguished in the emission of DsRed.

ous observations. ${ }^{[6]}$ The inequal reduction of the intensity upon photodissociation of one of the chromophores has been understood in terms of photogenerated traps which partially quench the fluorescence of the remaining subunits. ${ }^{[6]}$ Other photoinduced processes known to occur in autofluorescent proteins such as DsRed and GPP ${ }^{[5,21]}$ were minimized by the use of moderate excitation intensity, while yielding a significant number of coincidences in the interphoton time histogram. However, the photon emission rate of DsRed was insufficient to derive a coincidence histogram for each individual level of a DsRed tetramer. By taking full advantage of the single DsRed time traces, separate coincidence histograms 
have been constructed for each of the four intensity levels in all fluorescence trajectories. Of the 285 molecules analyzed, $14 \%$ show only one intensity level, $28 \%$ exhibit two levels, $34 \%$ three levels, and $24 \%$ of the molecules display four intensity levels, which is in satisfactory agreement with our previous findings. ${ }^{[6]}$

Figure 3 shows the individual coincidence histogram for each of the four intensity levels, together with the overall histogram. The characteristic pulsed antibunching feature, that is,

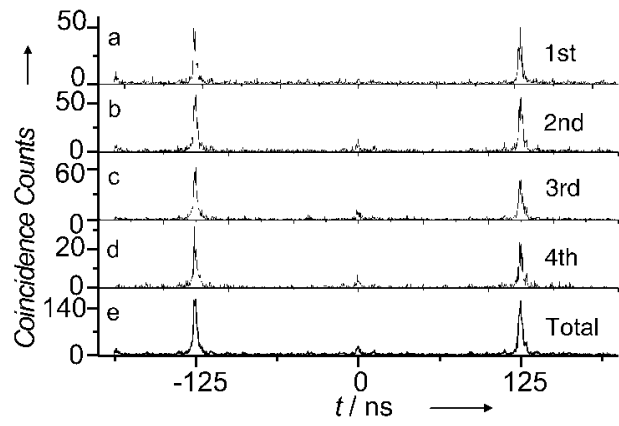

Figure 3. $a-d$ ) Interphoton times histogram (raw data) for the first (lowest intensity level) to the fourth (highest intensity level) levels and total histogram (e) of 285 molecules of DsRed obtained by adding all levels (from a to d).

photon coincidences around $\tau= \pm 125 \mathrm{~ns}$ and the lack of coincidences at $\tau=0$, is clearly evident for all of the intensity levels. Complete antibunching in the lowest level is consistent with the fact that only one active chromophore is present in the system. On the other hand, the strongly decreased coincidences at $\tau=0$ for the other three intensity levels is unexpected for a multiemitter system unless some degree of dipole coupling between the chromophores exists. From the antibunching histograms the ratio $N_{0} / N_{\mathrm{L}}$ between the area of the peak at $\tau=0, N_{0}$, and the mean value of the area of the lateral peaks, $N_{\mathrm{L}}$, can be used to estimate the number of independent emitters $(m)$ by using the relation $N_{0} / N_{\mathrm{L}}=1-(1 / m) .{ }^{[14]}$ Ideally, for one emitter the $N_{0} / N_{\mathrm{L}}$ ratio should be 0 , for two independent emitters 0.5 , etc. Table 1 shows the $N_{0} / N_{\mathrm{L}}$ ratios for independent emitters as well as the $N_{0} / N_{\mathrm{L}}$ as obtained from our histograms. The $N_{0} / N_{\mathrm{L}}$ ratios for all four levels in the DsRed remain close to zero and considerably lower than 0.5 , which indicates that the emission from DsRed has predominantly a single-molecule character and points to excitonic coupling between the chromophores, as discussed below. The slight deviation of $N_{0} /$ $N_{\mathrm{L}}$ from zero $(0.10 \pm 0.03)$ for the lowest intensity level is due to background coincidence.

Table 1. $\mathrm{N}_{0} \mathrm{~N}_{L}$ ratios as expected for four independent emitters and the $\mathrm{N}_{0}$ $\mathrm{N}_{L}$ as derived from the photon-arrival-time histograms on DsRed

\begin{tabular}{|lll|} 
Number of absorbers & $N_{0} / N_{\mathrm{L}}$ (independent emitters) & $N_{0} / N_{\mathrm{L}}$ (exptl.) \\
\hline 1 & 0 & $0.10 \pm 0.03$ \\
2 & 0.5 & $0.14 \pm 0.03$ \\
3 & 0.67 & $0.16 \pm 0.04$ \\
4 & 0.75 & $0.19 \pm 0.07$ \\
\hline
\end{tabular}

We have also observed a modest but consistent increase of $N_{0} / N_{\mathrm{L}}$ for the upper intensity levels. This is a sign of multiple photon emission arising either from oligomers once in a while or continuously occurring on a minor subpopulation of the DsRed tetramers. In fact, from the difference between the $N_{0} /$ $N_{\mathrm{L}}$ (0.19) value for the highest intensity level and $N_{0} / N_{\mathrm{L}}(0.10)$ for the lowest level, we can make a first estimation of the occurrence of multiple photon emission. In the simplest case, assuming that the four subunits comprising the tetramers emit in an independent fashion, we would expect an $N_{0} / N_{\mathrm{L}}$ ratio of 0.75 (see Table 1). Thus, the percentage of multiple photon emission with four independent emitters would be: $(0.19-$ $0.10) / 0.75=12 \%$. At the other extreme, assuming that emission from the highest level in the tetramer occurs exclusively from double emitters, the percentage of multiple photon emission would be $18 \%$ (where $N_{0} / N_{\mathrm{L}}$ for two independent emitters is 0.5 ). In reality, the percentage of multiple photon emission will be somewhere in between these two values.

Taking into account the low signal-to-background $(S / B)$ ratio for DsRed, larger values for the $N_{0} / N_{\mathrm{L}}$ ratio would be expected even for a single emitter. ${ }^{[22]}$ However, the antibunching histogram for the background shows a rather flat distribution, which indicates a random arrival of background counts with no particular correlation to the laser pulse positions (data not shown). A reduced contribution of zero delay coincidences due to background to the $N_{0} / N_{\mathrm{L}}$ ratios is thus obtained. For the upper intensity levels, the influence of background is even less important as the $S / B$ increases, thereby supporting our assignment that the somewhat higher values of $N_{0} / N_{\mathrm{L}}$ obtained for the upper levels are due to a minor multiemitting contribution.

Both, strong (coherent) and weak (incoherent) excitonic coupling between the chromophores could be equally responsible for the antibunching histograms obtained. The first scenario is hardly plausible at room temperature when the interchromophoric distances and their respective dipole orientations are considered. ${ }^{[8,9]}$ Moreover, it has been demonstrated that the origin of the red shift in DsRed is related to the structure of the chromophore itself and not as a result of coherent excitonic coupling. ${ }^{[23]}$ On the other hand, incoherent excitonic coupling (Förster-type energy transfer) might efficiently occur, if we consider the DsRed geometry structure. ${ }^{[24]}$ The monomers in the tetramer are placed in a rectangular array (see Figure 1) with distances of $2.2,3.8$, and $4.3 \mathrm{~nm}$ between monomer pairs and displaying estimated angles of 21,47 , and $41^{\circ}$ between the monomer pairs $A-B$ and $C-D, A-C$ and $B-D$, and the diagonal pairs $A-D$ and $B-C$, respectively. ${ }^{[8,9]}$ Calculations taking into account such geometry indicate energy-transfer rates of 1.7.10 11 and $3.0 \cdot 10^{9} \mathrm{~s}^{-1}$ between monomer pairs $A-B, C-D$ and $A-C, B-D$ respectively, ${ }_{1}^{[7]}$ which are much faster than the fluorescence rate of $2.7 \times 10^{8} \mathrm{~s}^{-1}$.

A similar type of Förster energy transfer will occur when two or more subunits in the tetramer are simultaneously excited to their first singlet state $\left(S_{1}\right)$ : Energy transfer will take place between two excited chromophores, one unit relaxing back to the ground state $\left(S_{0}\right)$ and the other going through an energyup-conversion process to an upper energy level $\left(S_{n}\right)$. Intramo- 
lecular radiationless relaxation to the lowest $S_{1}$ level will rapidly follow from where ultimately a unique photon is emitted. These two steps, that is, energy up-conversion mediated by Förster-type energy transfer, and fast intramolecular relaxation to the lowest $S_{1}$ state represent singlet-singlet annihilation (SSA), which prevents the system from residing in a doubly excited state with both subunits in the $S_{1}$ state. ${ }^{[16]}$ We favor this process to explain the single emitter behavior of DsRed. Singlet-singlet annihilation has been also proposed as the process responsible for the antibunching behavior observed in artificial multichromophoric systems. ${ }^{[15-17]}$ It is important to notice that the efficiency of this process is highly dependent on the spectral overlap of the emission and absorption spectra of the transitions involved, that is, the $S_{1} \rightarrow S_{0}$ fluorescence and the $S_{1} \leftarrow S_{0}$ and $S_{n} \leftarrow S_{1}$ absorptions. SSA will only be accomplished if the transfer rates between excited states are faster than the fluorescence rate. All spectra except the $S_{n} \leftarrow S_{1}$ absorption spectrum are known for DsRed, yielding transfer rates one to three orders of magnitude faster than the fluorescence rate, as indicated above. Although spectral data on the $S_{n} \leftarrow S_{1}$ transition is not yet available, it is known that for most organic dyes this transition is slightly lower in energy that the $S_{1} \rightarrow S_{0}$ transition, with a resulting large spectral overlap between the $S_{1} \rightarrow S_{0}$ and $S_{n} \leftarrow S_{1}$ transitions, which supports our interpretation of the antibunching data.

However, the slight increase of $N_{0} / N_{\mathrm{L}}$ with the intensity levels might be indicative of a subpopulation of the chromophores showing little or no coupling and leading to multiple photon emission. Numerical simulations on a donor-acceptor dye pair have shown that stochastic variations in the donoracceptor coupling strength can account for a loss of excitonic coupling in the system. ${ }^{[25]}$ In our case, variations of the coupling strength may be due to minor rearrangements of the subunits in the oligomer as a result of the confinement in the gel and/or to changes in the spectral overlap between donor and acceptor caused by DsRed photoconversion. ${ }^{[5]}$ These variations may result in a decrease of efficiency of the energy-transfer processes.

In conclusion, we have used pulsed photon antibunching at room temperature to uncover the single-quantum-emitting character in a naturally occurring multichromophoric system, the red autofluorescent protein, DsRed. By disentangling all emission levels, we have demonstrated that the fluorescence emission in the DsRed tetramer proceeds dominantly from only one emitter at a time, thereby confirming its weak excitonic coupling character. Differences in coupling strength leading to loss of excitonic coupling and multiple photon emission were directly observed from the antibunching histograms. Our experimental and data analysis approaches have potential for the investigation of conformational fluctuations and intermolecular interactions of complex (bio)systems at room temperature.
Research on Matter (FOM) which is financed by the Netherlands Scientific Organization (NWO), EU Madam Curie Fellowship (HPMF-CT-2002-01698) and the Royal Netherlands Academy of Arts and Sciences (KNAW).

Keywords: excitonic interactions - FRET (fluorescence resonance energy transfer) $\cdot$ photon antibunching $\cdot$ proteins. single-molecule studies

[1] R. Y. Tsien, Annu. Rev. Biochem. 1998, 67, 509.

[2] M. V. Matz, A. F. Fradkov, Y. A. Labas, A. P. Savitsky, A. G. Zaraisky, M. L. Markelov, S. A. Lukyanov, Nat. Biotechnol. 1999, 17, 969.

[3] G. S. Baird, D. A. Zacharias, R. Y. Tsien, Proc. Natl. Acad. Sci. USA 2000, 97, 11984.

[4] R. E. Campbell, O. Tour, A. E. Palmer, P. A. Steinbach, G. S. Baird, D. A. Zacharias, R. Y. Tsien, Proc. Natl. Acad. Sci. USA 2002, 99, 7877.

[5] M. Cotlet, J. Hofkens, S. Habuchi, G. Dirix, M. Van Guise, J. Michiels, J. Vanderleyden, F. C. De Schryver, Proc. Natl. Acad. Sci. USA 2001, 98, 14398.

[6] M. F. Garcia-Parajo, M. Koopman, E. M. H. P. van Dijk, V. Subramaniam, N. F. van Hulst, Proc. Natl. Acad. Sci. USA 2001, 98, 14392.

[7] B. Lounis, J. Deich, F. I. Rosell, S. G. Boxer, W. E. Moerner, J. Phys. Chem. B. 2001, 105, 5048 .

[8] M. A. Wall, M. Socolich, R. Ranganathan, Nat. Struct. Biol. 2000, 7, 1133.

[9] D. Yarbrough, R. M. Wachter, K. Kallio, M. V. Matz, S. M. Remington, Proc. Natl. Acad. Sci. USA 2001, 98, 462.

[10] L. Mandel, E. Wolf, Optical Coherence and Quantum Optics. Cambridge University Press, New York, 1995.

[11] T. Basché, W. E. Moerner, M. Orrit, H. Talon, Phys. Rev. Lett. 1992, 69, 1516.

[12] L. Fleury, J.-M. Segura, G. Zumofen, B. Hecht, U. P. Wild, Phys. Rev. Lett. 2000, 84, 1148.

[13] B. Lounis, W. E. Moerner, Nature 2000, 407, 491.

[14] P. Tinnefeld, C. Muller, M. Sauer, Chem. Phys. Lett. 2001, 345, 252.

[15] P. Tinnefeld, K. D. Weston, T. Vosch, M. Cotlet, T. Weil, J. Hofkens, K. Müllen, F. C. De Schryver, M. Sauer, J. Am. Chem. Soc. 2002, 124, 14310.

[16] C. G. Hübner, G. Zumofen, A. Renn, A. Herrmann, K. Müllen, T. Basché, Phys. Rev. Lett. 2003, 91, 093903.

[17] J. Hofkens, M. Cotlet, T. Vosch, P. Tinnefeld, K. D. Weston, C. Ego, A. Grimsdale, K. Müllen, Proc. Natl. Acad. Sci. USA 2003, 100, 13146.

[18] C. Hettich, C. Schmidt, J. Zitzmann, S. Kühn, I. Gerhard, V. Sandoghdar, Science 2002, 298, 385.

[19] J. Hernando, M. van der Schaaf, E. M. H. P. van Dijk, M. Sauer, M. F. Garcia-Parajo, N. F. van Hulst, J. Phys. Chem. A. 2003, 107, 43.

[20] R. Hanbury-Brown, R. Q. Twiss, Nature 1956, 177, 27.

[21] M. F. Garcia-Parajo, G. M. J. Segers-Nolten, J. A. Veerman, J. A. Greve, N. F. van Hulst, Proc. Natl. Acad. Sci. USA 2000, 97, 7237.

[22] K. D. Weston, M. Dyck, P. Tinnefeld, C. Müller, D. P. Herten, M. Sauer, Anal. Chem. 2002, 74, 5342.

[23] S. Boyé, H. Krogh, I. B. Nielsen, S. B. Nielsen, S. U. Pedersen, U. V. Pedersen, L. H. Andersen, A. F. Bell, X. He, P. J. Tonge, Phys. Rev. Lett. 2003, 90, 118103.

[24] T. A. Schüttrigkeit, U. Zachariae, T. von Feilitzsch, J. Wiehler, J. von Hummel, B. Steipe, M. E. Michel-Beyerle, ChemPhysChem 2001, 2, 325.

[25] A. J. Berglund, A. C. Doherty, H. Mabuchi, Phys. Rev. Lett. 2002, 89, 068101.

Received: May 7, 2004

Revised: August 23, 2004

\section{Acknowledgements}

Samples were a kind gift of V. Subramaniam. This work was financially supported by the Dutch Foundation for Fundamental 